\title{
Lys van Keurders 2008
}

Prof D (Dan) Baird, Stellenbosch Universiteit

Prof. WS (Willem) Boshoff, Universiteit van Suid-Afrika, Pretoria

Prof H H (Hein) du Preez, Wetenskaplikedienste Rand Water.

Prof Dr Herman Eijsackers, Wageningen University and Research Centre/

Vrije Universiteit Amsterdam

Mnr PSO (Paul) Fouche, Universiteit van Venda, Louis Trichardt

Dr L C (Leon) Geustyn, GLS Consulting (Pty) Ltd

Mev ML (Mariejtie) Griffioen, Universiteit van Johannesburg

Prof JT (Tertius) Harmse, Universiteit van Johannesburg

Prof PS (Stephan) Heyns, Universiteit van Pretoria

Prof TE (Theo) Kleynhans, Stellenbosch Universiteit

Me R (Rose) Luke, Universiteit van Johannesburg

Prof Mark Maboeta, Noordwes-Universiteit, Potchefstroom

Prof. Miles B. Markus, Universiteit van die Witwatersrand, Johannesburg.

Dr JP (James) Odendaal, Kaapse Skiereilandse Universiteit van Tegnologie, Kaapstad

Prof Annemarié Avenant-Oldewage, Universiteit van Johannesburg

Prof. Herman Potgieter, Manchester Metropolitan University

Prof L (Laurette) Pretorius, Universiteit van Suid-Afrika, Pretoria

Prof LM (Lou) Pretorius, Universiteit van Pretoria

Prof AJ Reinecke, Stellenbosch Universiteit

Prof SA (Sophiè) Reinecke, Stellenbosch Universiteit

Dr E Roekens, Vlaamse Milieumaatschappij

Prof AA (Andries) Stulting, Universiteit van die Vrystaat, Bloemfontein

Dr E (Lize) Theron. Sentrale Universiteit vir Tegnologie, Vrystaat

Prof AJ (Abrie) van der Merwe, Universiteit van die Vrystaat, Bloemfontein

Dr Esbeth van Dyk, CSIR Built Environment, Stellenbosch

Prof M (Retha)Viljoen, Universiteit van Pretoria 